\title{
Approche par compétences, définition et désignation des savoirs mathématiques : peut-on envisager la disparition d'une organisation disciplinaire des savoirs?
}

Approaches by competences, definition and designation of the mathematical knowledge: can we consider the disappearance of a disciplinary organization of knowledge?

\section{Maggy Schneider et Alain Mercier}

\section{(2) OpenEdition}

\section{Journals}

Édition électronique

URL : https://journals.openedition.org/educationdidactique/1967

DOI : 10.4000/educationdidactique.1967

ISSN : 2111-4838

Éditeur

Presses universitaires de Rennes

Édition imprimée

Date de publication : 12 décembre 2014

Pagination : 109-124

ISBN : 978-2-7535-39-66-2

ISSN : 1956-3485

Référence électronique

Maggy Schneider et Alain Mercier, « Approche par compétences, définition et désignation des savoirs mathématiques : peut-on envisager la disparition d'une organisation disciplinaire des savoirs? ", Éducation et didactique [En ligne], 8-2 | 2014, mis en ligne le 12 décembre 2016, consulté le 22 juillet 2022. URL : http://journals.openedition.org/educationdidactique/1967 ; DOI : https://doi.org/10.4000/ educationdidactique.1967 


\title{
APPROCHE PAR COMPÉTENCES, DÉFINITION ET DÉSIGNATION DES SAVOIRS MATHÉMATIQUES : PEUT-ON ENVISAGER LA DISPARITION D'UNE ORGANISATION DISCIPLINAIRE DES SAVOIRS?
}

\author{
Maggy Schneider, Université de Liège \\ Alain Mercier, EAM ADEF, Université de Provence, IFE ENS-Lyon ${ }^{1}$
}

\begin{abstract}
Linsistance sociale à réformer les programmes d'enseignement en décrivant les compétences que les élèves doivent acquérir et non plus les savoirs que les professeurs doivent enseigner n'est pas seulement l'effet d'une demande de pilotage de l'enseignement par l'évaluation. La question du savoir considéré comme pouvoir d'agir dans le monde y est apparemment centrale et suppose que l'agir soit l'effet de la résolution d'un problème, action raisonnable. Les auteurs montrent ici comment les problématiques didactiques permettent de définir les conditions nécessaires à la réussite d'un enseignement «à (et par) la résolution de problèmes ». Plus particulièrement, ils illustrent que l'analyse des enjeux de savoir de cette résolution souligne la nécessité d'étudier les classes de problèmes que le savoir permet de résoudre. Les exemples analysés sont relatifs à l'approche par compétences en Communauté française de Belgique (CFWB) mais nous avons tout lieu de penser qu'ils sont caractéristiques de cette mouvance pédagogique en d'autres régions du monde.
\end{abstract}

Mots-clés : Savoirs, compétences, didactique, apprentissage des savoirs, approche par compétences, apprentissage à la résolution de problèmes, classes de problèmes, systèmes de représentation, jeux de langage.

Approaches by competences, definition and designation of the mathematical knowledge : can we consider the disappearance of a disciplinary organization of knowledge?

The social insistence to reform teaching curricula by describing competences that the students must acquire, instead of describing the knowledge that must be taught, is not the sole effect of a request for piloting education by evaluation. The issue of knowledge being considered as capacity to act in this world is apparently central herein, and supposes that acting be the effect of solving a problem. The authors then show how the didactical problematics allows to define the necessary conditions for the success in teaching " to solve problems " (and problem solving). Especially, they illustrate that the analysis of the knowledge stakes for this resolution stresses the need to study the classes of problems that knowledge solves. The discussed examples are related to the competency-based approach in the French Community of Belgium (CFWB) but we have reasons to think that they are characteristic of this educational movement in other parts of the world.

Keywords: Knowledge, competences, didactics, knowledge learning, approach by competences, learning by problem solving, classes of problems, representation systems, language play. 
Ce texte revisite l'articulation entre, d'une part, « l'apprentissage à la résolution de problèmes » ainsi appelé et promu dans « l'approche par compétences » (APC) et, d'autre part, l'apprentissage des savoirs mathématiques. Le regard porté est outillé d'analyses didactiques publiées, prenant appui sur la théorie des situations de Brousseau (TSD) en articulation avec la théorie anthropologique du didactique de Chevallard (TAD), mais aussi sur une étude épistémologique du fonctionnement, différencié d'une discipline à l'autre, de compétences supposées transversales. Ces analyses crédibilisent une façon d'envisager l'apprentissage à la résolution de problèmes et la question liée du transfert des savoirs. Nous y reviendrons plus loin et la résumons brièvement comme suit. Les savoirs mathématiques sont modélisés par des classes de problèmes, mais l'exercice de la résolution de problèmes y est structuré par ces mêmes classes, l'enjeu n'étant pas la résolution de problèmes quelconques mais bien quelques techniques de résolution des problèmes d'une même classe. Nous montrerons que les outils pédagogiques destinés aux enseignants en Belgique francophone, en réponse au malaise créé par le paradigme des compétences, sont propres à occulter des gestes d'étude et, par-là, des gestes d'enseignement que supposent la désignation d'une classe de problèmes et l'intelligibilité que les élèves peuvent en avoir. À travers ces exemples, notre intention est de donner à comprendre les difficultés que pose à l'enseignement le mot d'ordre « enseigner pour former des compétences » vécu sous un mode idéologique, que ce soit dans cette région ou une autre.

\section{QUELQUES TRAITS SIGNIFICATIFS DE L'APPROCHE PAR COMPÉTENCES : L'EXEMPLE ÉCLAIRANT DE LA CFWB}

Cette section prend largement appui sur plusieurs de nos publications antérieures. Il nous paraît important de reprendre ici des conclusions de ces travaux susceptibles d'expliquer le succès du paradigme des compétences, en Belgique ou ailleurs, et son fonctionnement essentiellement idéologique difficile à dénaturaliser car il semble proposer une solution aux problèmes soulevés par l'organisation scolaire de la transmission des œeuvres culturelles. Pour les données empiriques et de plus amples analyses, nous renvoyons le lecteur à ces références ou à d'autres mentionnées ci-après, nous contentant de préciser, à grands traits, l'hypothèse dégagée qui éclairera la suite de notre article. Cette hypothèse résulte d'un faisceau de faits et d'analyses d'origines diverses: histoire et sociologie de l'éducation, étude des systèmes éducatifs, analyse didactique de dispositifs de formations.

En $\mathrm{CFWB}^{2}$, l'Approche Par Compétences fait l'objet, depuis plus une quinzaine d'années, d'un discours d'intention sur les missions de l'enseignement (Décret «Missions », 1997). Comme ailleurs, on ne peut nier l'influence du monde de l'entreprise sur l'avènement des compétences à l'école, influence qui fait aujourd'hui l'unanimité et que De Ketele (2003) explique en substance comme suit. Devant souscrire aux impératifs de la rentabilité et de la compétitivité et constatant une certaine inefficacité des nouveaux diplômés à être rapidement performants, les entreprises sont amenées à créer leurs propres formations axées sur des référentiels de compétences des métiers. Toutefois, on s'aperçoit vite que des postes de niveau plus élevé requièrent des compétences transversales ou génériques telles que « interpréter correctement un problème », " réagir de façon critique à une situation $», \ldots$ dont l'exercice serait fort coûteux si elle était à charge des entreprises. De là l'idée de faire pression sur les autorités des systèmes éducatifs pour insérer l'apprentissage de telles compétences dans les programmes d'étude.

Mais d'autres circonstances peuvent expliquer le succès de l'APC. Ainsi, en ce qui concerne la CFWB, Draelants et al. (2003) observent que deux grands débats animent le monde scolaire dans la période durant laquelle cette réforme s'est mise en place : la question du financement de l'enseignement, d'une part, et celle des objectifs et du fonctionnement du système, d'autre part. L'APC devient alors, tant pour les politiciens que pour les membres de la noosphère, le fer de lance d'un discours sur les finalités de l'enseignement, discours dont le caractère idéologique se mesure à l'empressement d'en évaluer les effets avant même d'avoir approfondi la réflexion sur les moyens (Gerard et Van Lint, 2000). On observerait donc ici un discours volontariste dont la fonction serait d'apaiser les esprits. Encore fallait-il le rendre crédible car imposer une réforme aux enseignants sans leur fournir le surcroît de moyens qu'ils revendiquent suppose de la justifier par des arguments plus « politiquement corrects » que les seules références au monde socio-économique, c'est-à-dire par des arguments pédagogiques. C'est le phénomène de « solipsisme en pédagogie » ainsi nommé par Crahay 
et Forget (2006) qui empêcherait, selon eux, que les pôles pédagogique et sociétal interagissent en favorisant une « conception linéaire et technocratique de la construction des curriculums : les enjeux sociétaux une fois réglés, les objectifs sont confiés aux techniciens du curriculum qui se chargent de leur opérationnalisation pédagogique $»$.

Ce sont les théories socioconstructivistes qui sont avancées comme arguments. Mais, comme le développe Schneider (2006a, 2006b, 2007), plusieurs écrits (entre autres Jonnaert et Vanderborght, 1999; Roegiers, 2000) qui soutiennent ainsi cette réforme des compétences convertissent indûment des théories d'apprentissage, modèles du développement mental de l'enfant, en mots d'ordre pour l'action enseignante au sein d'institutions scolaires. Les dites situations-problèmes deviennent alors les emblèmes de dispositifs de formation ou d'évaluation formative visant à entraîner les élèves, sur la base d'aspects purement méthodologiques, à de supposées compétences générales comme l'autonomie et la créativité, transversales comme formuler une hypothèse et communiquer, ou plus précises mais non moins abstraites comme interpréter correctement un problème (i.e. : son énoncé ?), toutes interprétées uniformément d'une discipline à l'autre faute d'une analyse épistémologique, comme celle de Schneider (2004), mettant en évidence leurs particularités au sein de chacune. On peut par ailleurs avancer que le mythe du transfert des compétences (implicitement associé à la mobilité des travailleurs) explique l'accent mis sur les compétences transversales.

Aussi, les situations-problèmes pratiquées sous l'égide de l'APC pêchent souvent par leur absence de caractère fondamental relativement à un savoir visé, soit parce qu'il n'y a tout simplement pas de savoir en jeu, soit parce qu'il y a en a bien un mais que, pour les élèves considérés, il ne constitue pas la réponse optimale à la question qui leur est dévolue (Schneider et Mercier, 2008). Elles ne respectent pas non plus des conditions, formulées tant en termes d'existence de milieu adidactique que de nécessité d'institutionnalisation, sans lesquelles ne peut s'envisager une quelconque dévolution d'une question aux élèves. Dans cette perspective, l'utilisation de la notion de situation - telle que pratiquée lors des formations des conseillers pédagogiques ou observée dans les manuels scolaires, voire les documents d'accompagnement des programmes (Schneider, 2007 et 2011a) - est à l'envers des résultats apportés par la recherche en didactique : elle fait fi des conditions sous lesquelles l'activité des élèves, en situation, est a priori susceptible de conduire à l'apprentissage de savoirs mobilisables dans une autre situation.

Ainsi l'entraînement à la résolution de problèmes devient, avec l'APC, à la fois la fin et le moyen : elle se dit d'ailleurs "Approche PAR Compétences» alors que "Approche POUR les Compétences» rendrait mieux compte d'un discours sur les seules finalités. On voit donc à l'œuvre un mécanisme dont nous faisons l'hypothèse qu'on peut en observer des composantes analogues ailleurs qu'en CFWB : dans un arrière-fond de pressions sociales sur le monde de l'enseignement, naît un discours politique sur les finalités de celui-ci, une idéologie au sens assez louable que lui donne Baechler (1976) laquelle « veut réaliser une valeur par l'exercice du pouvoir dans une société »; une conjoncture de crise économique la convertit malheureusement en idéologie au sens péjoratif que lui donnait Lalande (2002) : «Pensée détachée du réel, qui se développe abstraitement sur ses propres données, mais qui est en réalité l'expression de faits sociaux, particulièrement de faits économiques, dont celui qui la construit n'a pas conscience, ou du moins dont il ne se rend pas compte qu'ils déterminent sa pensée ». Et sans doute est-ce là ce qui peut expliquer le bien-fondé d'un jugement porté par Crahay (2006) qui n'hésite pas à parler de doxa à propos de la réforme des compétences.

On observe alors - et nous y arrivons - que les problèmes porteurs de ces supposées compétences posent, aux élèves comme à leurs enseignants, des difficultés persistantes. Sans doute, il pouvait y avoir quelque pertinence à tenter de redéfinir l'organisation des savoirs en disciplines. Cette organisation leur a trop souvent aujourd'hui fait perdre leur propriété première de pouvoir d'agir dans le monde, sans que l'on ne sache bien pourquoi sinon qu'il s'agit d'un problème de transposition didactique ${ }^{3}$. Mais nous observons que la transposition de la résolution de problèmes produit l'oubli des compétences initialement et officiellement visées. Celles-ci sont bientôt remplacées, soit par une décomposition des difficultés en niveaux de tâches permettant d'adapter l'évaluation à la compétence des élèves (et à renoncer à l'enseignement des compétences de haut niveau), soit par l'enseignement d'un supposé processus de résolution permettant en principe la réussite des élèves... tant que les problèmes posés relèvent du processus enseigné. C'est ce que nous développons ci-dessous. 


\section{DEUX ATTITUDES CONTRASTÉES FACE AUX DIFFICULTÉS DES ÉLÈVES À RÉSOUDRE LES PROBLÈMES}

Lors des formations et d'enquêtes non publiées organisées par les réseaux publics d'enseignement, les professeurs de mathématiques témoignent de leurs difficultés à " faire apprendre à leurs élèves à résoudre des problèmes $»$ (Schneider, 2011a). Devant les échecs répétés de ceux-ci lors des évaluations, ils se replient peu à peu sur des évaluations qui font la part belle aux acquisitions techniques ou à un bachotage caché des problèmes précédemment posés lors des évaluations officielles.

Un repli analogue s'observe chez des chercheurs en sciences de l'éducation auxquels la CFWB a donné mandat pour concevoir des épreuves d'évaluation des compétences auprès d'une cohorte importante d'élèves concernés par le socle commun. C'est le cas de Rey (2009) et Kahn (2010) dont nous résumons ici la position commune élaborée au sein de la même équipe. Tous deux commencent par distinguer les «procédures » qui se ramènent à l'exécution d'une tâche relativement stéréotypée telle que Effectuer à la main un calcul isolé sur des nombres en écriture décimale de taille normale et les « compétences avec mobilisation », c'est-à-dire des « compétences qui impliquent que l'élève doive choisir, parmi les procédures qu'il connaît, celle ou celles qu'il y a lieu de mettre en œuvre dans une situation nouvelle ». Affirmant en conséquence que "la compétence avec mobilisation ne saurait être attestée que par l'affrontement de l'élève à une situation inédite », ils proposent alors

la passation des épreuves d'évaluation, dans chaque classe, en trois temps répartis sur la semaine :

d'abord la situation complexe qui requiert la mise en œuvre et la combinaison de plusieurs procédures ;

ensuite, dans un second temps, cette situation découpée en «petits problèmes » qui nécessitent la mobilisation d'une seule procédure ;

enfin, ce sont des batteries d'exercices correspondants aux procédures requises dans les deux temps précédents qui sont présentées aux élèves. (Kahn, 2010).

Pour Kahn (2010), une telle forme d'évaluation :

permet d'abord de donner à chaque élève toutes les chances de faire prendre en compte ce qu'il sait faire : la mobilisation complexe s'il le peut et, s'il ne le peut, la mobilisation simple et enfin s'il n'y arrive pas, on lui donne au moins la possibilité de montrer qu'il a automatisé certaines opérations élémentaires. (page 5 de la version électronique).

Ces chercheurs insistent sur le fait que la difficulté majeure est de faire partager aux élèves « le mode d'interprétation des tâches et des situations qui est celui de l'École » et motivent leur proposition en développant que le «à bon escient » dont il faut savoir faire preuve pour mobiliser les savoirs pertinents dans une situation donnée « ne s'enseigne pas ». Nous discuterons plus loin ce point de vue.

Une autre position, plus optimiste, est celle de Fagnant et Demonty (2005), autres chercheurs en sciences de l'éducation, qui signent, dans le cadre d'une recherche également commanditée par la CFWB, des guides méthodologiques à l'adresse des enseignants du primaire portant le titre significatif : "Résoudre des problèmes : pas de problème! ". Dans ces guides qui alimentent actuellement les formations d'enseignants, ces chercheuses visent à favoriser chez les élèves une démarche réflexive de résolution de problèmes en articulant deux objectifs : " développer chez les enfants des compétences propres à chaque phase du processus de résolution » et « contrecarrer les stratégies superficielles peu compatibles avec la mise en œuvre d'une démarche générale de résolution ».

Conformément au premier objectif, les problèmes multiples repris dans ces guides sont groupés en chapitres et sections qui correspondent aux étapes et démarches de la résolution de problèmes telles que mises en évidence par les psychologues cognitivistes, tels Schönfeld (1989) : d'abord, la représentation du problème et ce qu'elle suppose en termes, par exemple, d'estimation de la solution; ensuite, la résolution proprement dite du problème qui requiert de développer des « démarches de type essaiserreurs » et, parfois, de " décomposer le problème en sous-problèmes »; enfin, l'interprétation de la solution, y compris dans des situations " ouvertes", et la communication de celle-ci "sous une forme adaptée au contexte ». On ne dit pas pour autant ce qui s'apprend d'une démarche de type essais-erreurs qui décrit aussi bien le comportement d'un mathématicien s'engageant dans la première étude d'une question nouvelle que celui des mouches cherchant à sortir et rencontrant la vitre d'un vantail de fenêtre dont l'autre vantail peut être ouvert. On ne 
dit pas non plus comment l'enchaînement des sousproblèmes d'un problème donné peut être produit. Les ressorts majeurs de ces guides sont donc d'ordre dit « méthodologique » et concernent prioritairement les stratégies générales de résolution de problèmes scolaires (relevant d'un contrat didactique supposé pérenne) même si, sur les 280 pages que contient par exemple le guide écrit en 2005, 50 sont consacrées aux outils mathématiques spécifiques enseignés au niveau d'étude considéré : les grandeurs proportionnelles, les intervalles et les partages inégaux.

Quant au deuxième objectif, il conduit Fagnant et Demonty à choisir les problèmes proposés de manière à provoquer chez les élèves le « désapprentissage de stratégies superficielles et des présupposés associés ». Ces présupposés, selon Reusser et Stebler, 1997 ou Verschaffel et al., 2000, consistent, par exemple, à supposer que tous les problèmes proposés par les enseignants ou dans les manuels ont un sens, que tout problème a une solution et une seule et qu'elle doit se présenter sous une forme numérique et précise ou encore que la tâche peut être effectuée en exploitant les concepts et les formules qu'on vient d'apprendre. En clair, il s'agit de dénoncer le contrat didactique ordinaire, dont plusieurs auteurs (dont SchubauerLeoni, 1988) ont montré pourtant qu'il était à la source de tout apprentissage par enseignement : une telle position produit la perte de confiance des élèves envers leur professeur, et l'impossibilité d'enseigner.

Nous venons de décrire là deux positions contrastées : la première prévoyant un rabattement de l'évaluation de compétences complexes sur celle de savoirs élémentaires de type procédural, l'autre misant sur des compétences et stratégies transversales de résolution de problèmes. Ces deux positions, assez révélatrices, nous semble-t-il, de deux tendances observées à propos de l'APC que ce soit chez les enseignants ou les chercheurs, sont propres à occulter un autre regard qui suppose d'articuler compétences et savoirs et que nous décrivons ci-après.

\section{MODÉLISER LES PROBLÈMES PAR LES SAVOIRS, POUR ENSEIGNER : UNE PERSPECTIVE PEU VISIBLE}

Notre position découle d'un regard particulier sur la spécificité des savoirs comme connaissances déclarées et publiquement partagées, et en particulier des savoirs mathématiques dans la résolution de problèmes et la question du transfert qui y est relative. Elle est développée amplement dans Schneider (2006b et 2011b) sur base de la TSD et de la TAD. Nous prêtons aux savoirs une fonction essentielle dans la recherche d'une "économie de pensée » et d'action pour reprendre et étendre une expression de $\mathrm{Mach}^{4}$. Les savoirs « tuent» les problèmes en permettant de les traiter, par catégories, au moyen de techniques conviviales. La modélisation de leur production en termes de praxéologies, par Chevallard (1992 et 1999), permet de rendre compte de cette dynamique. S'y articulent en effet, d'une part, une dimension «praxis » qui met en correspondance des types de tâches et des techniques permettant de les effectuer et, d'autre part, le discours technologique ou théorique qui justifie et rend intelligible les techniques considérées eu égard aux tâches données : en somme, pour pouvoir «tuer » les problèmes, il faut en payer le prix en s'assurant de la pertinence des techniques utilisées pour les traiter. Cependant cette dynamique reste intimement liée au caractère fondamental, au sens de Brousseau (1973, 1986 et 1998), des tâches initiales définies. C'est pourquoi, nous restons attachés à l'idée que les savoirs sont modélisés par des situations fondamentales qui en restaurent les raisons d'être. Mais nous tenons aussi à l'idée que les problèmes sont modélisés par les savoirs - d'où notre titre - au sens où la résolution de problèmes, s'apprend, en mathématiques, par catégorisation de problèmes, les catégories faisant pendant à ces situations fondamentales (Schneider, 2006a et 200b) sans qu'il n'y ait cependant de correspondance biunivoque entre elles. En effet, la catégorisation des problèmes peut s'effectuer, soit à partir des types de tâches définies par une (ou des) situation(s) fondamentale(s), soit à partir des techniques vues comme méthodes spécifiques de résolution des problèmes d'une certaine classe. Ainsi, pour reprendre les exemples analysés ici, on peut parler des problèmes d'optimisation, ceux-ci pouvant se résoudre par la technique de dérivation mais aussi, dans certains cas, par la résolution d'inéquations, voire grâce à des méthodes géométriques. Mais on peut parler aussi des problèmes relevant du calcul des dérivées en groupant alors des tâches d'optimisation avec d'autres tâches liées à la détermination de vitesses ou à l'approximation affine locale de fonctions.

S'il s'agit bien d'apprendre à résoudre des problèmes, nous faisons donc l'hypothèse que la résolution de problèmes (et donc le « à bon 
escient ») s'enseigne, parce que les problèmes peuvent être organisés selon les techniques de leur résolution ou selon les situations fondamentales qui modélisent les savoirs associés. Mais cette position tranche, d'une part, par rapport à celle prise par Kahn et Rey et, d'autre part, par rapport à celle de Demonty et Fagnant, telles que décrites plus haut. La nôtre suppose en effet un dispositif didactique particulier, que nous décrirons et justifierons a contrario des pratiques observées. Convoquons pour ce faire le concept de famille de tâches introduit par Beckers (2002) pour traduire l'idée que plusieurs tâches peuvent avoir une parenté telle que le travail fait sur l'une d'elles favorise l'exécution d'une autre tâche de la même famille. Schneider (2006a) a montré qu'en CFWB de telles familles renvoient souvent aux étapes d'une démarche générale de résolution de problèmes : "se poser des questions ", "se documenter ", « formuler une hypothèse explicative », « rédiger une solution », ce qui fait penser à l'optique défendue par Demonty et Fagnant (2005). Or, comme développé par Schneider (2006b), rien ne laisse supposer, ni dans les recherches des didacticiens, ni dans celles des psychologues cognitivistes (Tardif, 1999) que ce soit là une piste intéressante. De même serionsnous dubitatifs sur une catégorisation des tâches à partir de présupposés des élèves sur les problèmes et les solutions attendues. Par contre, notre manière de voir les mathématiques nous suggère de constituer des familles en croisant, d'une part, des tâches porteuses de mathématiques et, d'autre part, des théories ou ensembles de résultats mathématiques et les techniques qu'ils autorisent. C'est ainsi que, à nos yeux, le théorème de Pythagore fédère l'ensemble des situations dans lesquelles il convient de l'appliquer et qui, au niveau d'étude considéré, relèvent de l'exploitation d'un triangle rectangle dont on connaît 2 côtés. On peut donc parler en ces termes de la famille des situations " Pythagore », ce que font Kahn (2010) et Rey (2009) sans pour autant la référer à l'ensemble des notions mathématiques qui lui sont associées.

Mais que peut-on espérer de ce concept de famille de situations? Les auteurs qui viennent d'être cités pensent qu'il conduit à de fausses pistes. Rey (Ib.) en identifie deux. D'abord

... le fait de croire qu'en renforçant l'automatisation des procédures, on va aider l'élève à être plus apte à les mobiliser à bon escient [...]
Ensuite

le fait de croire qu'on pourrait imaginer des familles de situations ou des familles de tâches qui permettraient qu'un élève familier d'un type de tâche puisse, parce qu'il sait dans quel type de tâche cette procédure est utilisée, la transférer dans un domaine proche.

Et de donner un exemple que Kahn (Ib.) explicite en ces termes:

On peut par exemple avoir expliqué à des élèves que le théorème de Pythagore est utilisable dans la famille des situations géométriques où l'on connaît la longueur de deux côtés d'un triangle rectangle et où on doit calculer celle du troisième ; pourtant, si l'énoncé du problème ne mentionne pas la présence d'un triangle rectangle (par exemple s'il est question de la diagonale d'un carré), certains élèves ne verront pas que le problème qu'ils ont à résoudre relève de cette famille.

Cet exemple nous donne l'occasion de préciser notre point de vue. Si nous y voyons que, pour partie, ces auteurs rejoignent notre façon de concevoir la notion de familles de situations, nous ne pouvonsnous empêcher de penser qu'ils ne voient pas la nécessité d'étudier avec les élèves la portée et les domaines d'usage des techniques. La détermination de sous-figures ou de sur-figures construites au prix du tracé ou de l'oubli d'une ligne supplémentaire est une technique générique en géométrie comme la production de sur formules ou de formules réduites est une technique algébrique : en oubliant deux côtés du carré on détermine un triangle rectangle où le théorème de Pythagore s'applique. On peut imaginer que les élèves n'y pensent pas a priori ou qu'ils ne s'y autorisent pas, mais on peut aussi montrer que cette technique s'enseigne et que les élèves peuvent devenir capables de la mobiliser d'eux-mêmes, dans d'autres cas. Ainsi pensons-nous que l'usage « à bon escient » s'enseigne : savoir est pouvoir agir, mais c'est d'abord pouvoir juger de l'action adéquate. Pour nous, savoir est donc une compétence technique forte.

À partir de là se profile une certaine façon d'intégrer les familles de situations dans l'enseignement. Elle est défendue par Schneider (2006b) en ces termes: " Plutôt que de penser ces familles comme un espace où le transfert devrait aller de soi ou peu s'en faut, il faudrait les considérer comme un objet d'enseignement, en apprenant aux élèves 
à distinguer ce qui rapproche les différentes situations de la famille mais aussi ce qui les distingue. » Prenons l'exemple des problèmes d'optimisation. Il ne suffit pas que les élèves aient résolu plusieurs de ces problèmes sous la guidance du professeur pour pouvoir gérer les suivants. Il faut aussi qu'ils les aient " étudiés » de sorte d'en identifier une trame commune : exprimer la grandeur à optimiser au moyen d'une fonction d'une variable indépendante, dériver cette fonction, annuler la dérivée et en déterminer le signe. Mais il faut aussi que l'élève voie la variabilité sous-jacente des paramètres qui définissent la famille. Dans certains problèmes, il n'y a qu'une seule variable indépendante, dans d'autres il y a deux variables liées. Il faut alors, dans ce dernier cas, exprimer l'une en fonction de l'autre, ce qui peut se faire de plusieurs manières suivant le contexte du problème : traduire des informations données dans l'énoncé (par exemple, le fait que telle grandeur est inversement proportionnelle à telle autre), ou exploiter, dans une figure donnée, des théorèmes de géométrie (souvent Thalès ou Pythagore), ou encore exprimer la constance d'un périmètre, d'une aire ou d'un volume. Et ce qui peut varier encore, c'est le type de fonction à dériver. Si les élèves n'apprennent pas à gérer cette variabilité propre à la famille de situations - variabilité définie a priori en fonction des objectifs de l'enseignement et du niveau d'étude - et/ ou si les questions d'évaluation débordent la famille ainsi circonscrite, il est vraisemblable, au vu des recherches en psychologie cognitive évoquées plus haut, que peu d'entre eux s'en sortent. Mais il faut aller plus loin encore et restaurer, dans l'enseignement, ce que Chevallard (1990) appelle une technique d'étude, c'est-à-dire

... non pas une procédure ou une méthode qu'il s'agit d'apprendre et de contrôler dans ses étapes, mais [ce qui] travaille la fonctionnalité d'un savoir dans la résolution d'un problème qu'il s'agit de roder dans des conditions standard, de tester dans des conditions limites, d'infirmer peut-être en bordure du champ (cité par Castella et Mercier, 1994).

Si le transfert des savoirs et techniques d'une situation à l'autre à l'intérieur d'une même famille doit faire l'objet d'un enseignement, a fortiori en est-il de tels transferts d'une famille à l'autre. La technique d'étude peut alors porter, suivant les cas, sur le choix d'une technique parmi plusieurs ou sur l'adaptation d'une technique à la tâche proposée. Ainsi, l'évaluation de grandeurs inaccessibles revient, bien souvent, à résoudre un triangle. Mais, suivant les tâches de cette famille, il faudra utiliser le théorème de Thalès, ou celui de Pythagore, ou un critère de similitude ou encore la trigonométrie. Quant à la technique de dérivation, elle doit être suivie d'une recherche de racines ou de celle d'images selon que l'on cherche à optimiser ou à déterminer une vitesse.

La position que nous défendons ici tient compte des aspects contractuels liés au transfert. Les chercheurs précédemment cités y sont sensibles: Kahn et Rey évoquent des difficultés qu'on peut interpréter en termes d'acculturation aux habitus du monde scolaire tandis que Fagnant et Demonty insistent sur les présupposés que les élèves peuvent avoir sur les problèmes travaillés à l'école. Bien avant, Castella et Mercier $(1994,1995)$ avaient, en ce qui concerne le transfert, fait l'hypothèse d'un fonctionnement sous contrat, au sens de Brousseau :

On peut dire que le contrat est rompu, pour un élève donné, lorsqu'il ne reconnaît plus ce qu'il devrait savoir faire : lorsque la faisabilité s'est perdue pour cet élève. La faisabilité se conserve tant que le problème est, au moins, reconnu [...]. Nous appelons plasticité $d u$ contrat la possibilité qu'il a de supporter cette variation, sa propriété de pouvoir varier autour de sa norme, dans un domaine où il sera reconnu comme identique à lui-même - ce qui garantit la faisabilité, par l'élève, des questions qui sont posées dans ce cadre. Une variation d'une composante du problème qui dépasserait le seuil de plasticité entraînerait la non faisabilité des questions obtenues, la rupture du contrat.

Pour eux, cette plasticité du contrat est différentielle suivant la position que l'élève occupe dans la hiérarchie de la classe et les « bons » élèves sont ceux qui font le plus les frais des ruptures de contrat signalées plus haut, sans doute pour avoir développé un rapport personnel trop conforme au rapport attendu par l'institution scolaire. Cette analyse permettrait de comprendre une observation de Perkins et Salomon (1989) selon laquelle les individus ne s'autorisent pas certains transferts. Effectivement le contrat didactique et les effets associés mettent bien en évidence que les comportements d'élèves sont dictés par ce qu'ils supposent que le professeur attend d'eux : souvent des réponses standard à des situations stéréotypées. C'est pourquoi, Schneider (2006b) propose 
de «mettre le transfert sous contrat » au sens du fonctionnement didactique décrit plus haut. Qu'il s'agisse d'apprendre à l'élève à manœuvrer à l'intérieur d'une famille de situations ou de lui apprendre à brasser plusieurs familles, le type de transfert est déclaré, « contractualisé », inscrit dans les objectifs : ainsi, on lui enseignera qu'il a à sa disposition plusieurs techniques, celles décrites plus haut, pour évaluer une grandeur inaccessible et que, en outre, il existe des critères de choix : par exemple, le fait de devoir gérer à la fois des longueurs et des amplitudes d'angles suggère d'utiliser des techniques trigonométriques. À charge pour l'élève de poursuivre l'étude qui, au départ des cas déjà rencontrés, lui permettra de gérer seul des situations nouvelles.

Dans notre perspective, un élève qui apprend à résoudre des problèmes, c'est donc avant tout un élève qui apprend à manœuvrer à l'intérieur de familles de situations au sens que nous lui donnons, décrit plus haut, et à brasser des familles de plus en plus nombreuses. Mais force est de constater que des dispositifs d'enseignement conçus dans cette perspective sont rarement organisés, dans les pratiques enseignantes, même au sein d'une seule année scolaire. Pas plus qu'ils ne sont envisagés dans les travaux des chercheurs belges en sciences de l'éducation ou les formations de formateurs qu'ils organisent. Schneider (2006b et 2011a) interprète ces observations par le fait que l'approche préconisée ici est moins glorieuse que l'intention portée par le discours idéologique, les transferts que l'élève arrive à faire ayant été en quelque sorte induits par l'enseignement, alors qu'on souhaite tester vraiment, sans concession, si les élèves sont capables de transférer une attitude "de recherche» ou une méthode générale de résolution de problèmes. Ainsi, des professeurs refusent d'enseigner à leurs élèves les structures mathématiques qui fédèrent en catégories les problèmes soumis aux élèves, afin de préserver leur caractère inédit et de pouvoir évaluer, de manière "scientifique ", la " compétence » de résolution de problèmes (Schneider, 2006a). Or, il y a bien nécessité d'un enseignement et c'est que développe la dernière section où nous montrons que la désignation des savoirs est une clé de leur transfert, non seulement au sein d'une même classe de problèmes, mais surtout d'une classe à l'autre. Nous le faisons au prix de certains développements mathématiques - mais des mathématiques élémentaires que tout un chacun a appris dans l'enseignement secon- daire - développements sans lesquels il ne peut y avoir description des enjeux de savoir, clé de toute didactique.

\section{DE LA DÉSIGNATION DES SAVOIRS MATHÉMATIQUES}

Dans les sections précédentes, nous avons développé à quel point la résolution de problèmes, démarche que l'APC présente comme essentielle, suppose la désignation de savoirs mathématiques. Rappelons que les situations fondamentales sont un modèle de ces savoirs, et même une forme de définition des problèmes respectifs que ces savoirs permettent de résoudre. Nous allons maintenant montrer comment c'est en définitive cette notion qui manque aux promoteurs d'un « enseignement à la résolution de problèmes ", quel est dans cette optique le processus de désignation des savoirs et ce qu'il suppose comme connaissances.

L'exemple retenu l'a été parce qu'il illustre particulièrement bien qu'il ne suffit pas de " décréter * l'importance de compétences transversales telles la résolution de problèmes "inédits » ou celle du transfert des savoirs au sein d'une discipline scolaire ou même d'une discipline à l'autre pour pouvoir observer, sur le terrain, des retombées positives de ce discours d'intentions louable en soi. Il s'agit effectivement ici, pour l'élève, de transférer le calcul des dérivées d'un problème d'optimisation dans un contexte physique familier à un autre dans un contexte géométrique nouveau. Les élèves sont ici en phase d'apprentissage et l'analyse montre, d'abord que ce transfert ne va pas de soi alors qu'il y est « sous contrat » au sens décrit plus haut, ensuite quels sont les gestes d'étude des élèves qui y arrivent et, par-là, quels pourraient être les procédés de désignation des savoirs décrits en didactique comme processus d'institutionnalisation. Mais, avant d'en venir à la situation elle-même et à son déroulement, nous précisons ses enjeux par les référents théoriques qui ont permis de la concevoir et de l'analyser.

Jusqu'à un certain point, cette situation relève d'un « jeu de cadres » au sens où l'entend Douady (1986) puisqu'elle invite les élèves à passer du cadre « cinématique " qui relève de la physique au cadre « géométrie » en mathématiques. Mais la définition même de cadre ne nous est pas tellement utile ici si ce n'est pour préciser que l'exemple décrit et analysé s'inscrit dans la construction d'un nouveau cadre 
appelé « analyse » au carrefour des deux précédents et, encore, dans sa forme embryonnaire qu'est le « calcul infinitésimal ». Aussi avons-nous choisi de caractériser la situation et l'ingénierie dont elle est issue en termes de ce que Schneider (2008) appelle des praxéologies 'modélisation'qu'elle distingue des praxéologies 'déduction'. Brièvement dit, les premières résultent d'un projet de modélisation d'objets non définis mathématiquement, existant par le truchement d'une désignation, mais dont on a une certaine connaissance : ce sont des 'préconstruits'au sens de Chevallard (1991). Les praxéologies 'déduction'relèvent, elles, d'un autre projet, à savoir l'organisation déductive des modèles construits dans les praxéologies 'modélisation', les objets devenant concepts mathématiques par le biais de définitions.

Si ce sont là deux projets complémentaires et fondateurs des mathématiques, il faut bien reconnaître que le projet de mise en forme déductive, le seul à conduire à des théories mathématiques au sens où l'entendent les mathématiciens, fait écran à l'autre pourtant fondamental dans une formation de base en mathématiques qui devrait en montrer le caractère instrumental y compris dans d'autres disciplines (Schneider, 2012). Et, souvent, le regroupement des problèmes en classes est évoqué à propos des concepts et théories mathématiques et envisagé à un niveau praxéologique qui n'est pas celui considéré ici. En effet, l'extrait d'ingénierie décrit ci-dessous s'inscrit dans une praxéologie 'modélisation'et nous l'illustrerons par la manière dont le concept de fonction y est traité. Lorsque certains chercheurs en didactique (Robert et Robinet, 1996) évoquent le caractère unificateur du concept de fonction, c'est le plus souvent par référence fut-elle implicite à la recherche de fondements des mathématiques et à leur structuration à partir des notions d'ensembles et de relations. Dans ce cadre, une relation fonctionnelle est définie en termes de triplets ce qui englobe aussi bien des transformations géométriques que les fonctions de l'analyse mathématique ou des opérateurs, tel celui de dérivation, qui agissent sur ces fonctions. Mais il existe, à propos des fonctions, un autre caractère unificateur qu'illustre bien le calcul intégral. Ainsi, au sens moderne de ce calcul, la quadrature d'un segment de parabole, la cubature d'une pyramide ou celle d'un cône appartiennent à la même catégorie de problèmes et même on peut dire qu'ils constituent un seul et même problème : ils sont tous modélisés en effet par l'intégrale définie d'une fonction du second degré et se résolvent par la primitivation d'une telle fonction. Un tel regard conduit à une classification des problèmes d'intégration suivant la nature de la « fonction-intégrande » sousjacente (fonction du $1^{\text {er }}$ degré, fonction inverse,...). Le caractère unificateur est ici algébrique et suppose non seulement une standardisation des variables indépendante et dépendante sous la forme $\mathrm{x}$ et y mais aussi le paramétrage des coefficients numériques qui va permettre d'adapter un modèle fonctionnel donné aux contraintes particulières du problème traité. Mais, ainsi que le montre notre exemple, cette unité fonctionnelle ne suffit pas pour fédérer divers problèmes en une même classe, le type de traitement fait aux fonctions concernées participant tout autant à cette catégorisation. En effet, les ostensifs algébriques ont pour nous un rôle important dans la classification des problèmes mathématiques car ils permettent des techniques de résolution très générales, telles que l'intégration ou la dérivation. L'extrait d'ingénierie décrit ci-dessous s'inscrit donc dans une praxéologie 'modélisation', niveau auquel nous considérerons l'économie de pensée offerte par les mathématiques. Dans ces conditions, enseigner « la résolution de problèmes » produit des savoirs techniques dans leur environnement praxéologique.

Les questions concernées sont issues d'une ingénierie didactique (Gantois et Schneider, 2012) dont la portée est essentiellement phénoménotechnique, visant à mettre en évidence les potentialités et les limites d'un milieu graphico-cinématique dans l'apprentissage des dérivées. Il s'agit de faire interpréter et étudier, du point de vue des vitesses, des mouvements rectilignes - dont certains ne sont pas uniformes - qui devront être précisés par les lois de mouvement. Avec des alea dont nous ne rendrons pas compte ici, ce travail débouche sur la formulation, au sein des classes, d'une vitesse instantanée comme « ce qu'il reste de l'expression d'une vitesse moyenne sur un intervalle $[\mathrm{t}, \mathrm{t}+\Delta \mathrm{t}]$ quand on rend $\Delta \mathrm{t}$ nul une fois faites les simplifications algébriques. » La vitesse instantanée est également interprétée comme pente du graphique de la loi de mouvement en un de ses points. Par contre, aucune règle de calcul de dérivation n'est institutionnalisée à l'étape dont nous ferons écho ici. Nous nous intéresserons en effet au moment où les chercheurs (et le professeur de la classe) essaient de faire évoluer la notion de vitesse instantanée en celle plus large de dérivée. Sans prétendre créer pour cela une situation à caractère adidactique, 
ils proposent aux élèves deux problèmes d'optimisation mobilisant une même fonction mais situés, le premier dans un contexte graphico-cinématique et le second dans un contexte géométrique.

Voici l'énoncé du premier problème :

Considérons un mobile sur une trajectoire rectiligne dont la loi de mouvement est donnée par la fonction $\mathrm{p}(\mathrm{t})=4\left(\mathrm{t}^{3}-25 \mathrm{t}^{2}+150 \mathrm{t}\right)$. Entre $\mathrm{t}=0$ et $\mathrm{t}=10$, à quel(s) instant(s) la distance du mobile à l'origine estelle maximale. Que vaut alors sa vitesse?

Et celui du second :

On considère à présent une plaque en tôle dont on a retiré les coins selon la figure suivante. En pliant les bords de cette plaque, on obtient une boîte rectangulaire et sans couvercle. Déterminer la valeur de x telle que le volume de la boîte soit maximum.

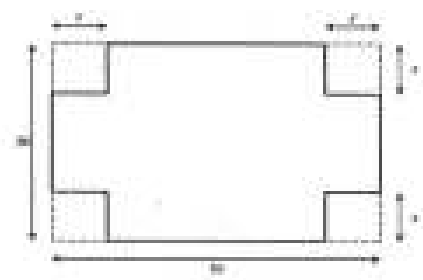

Ce dispositif est dicté par une observation antérieure (Schneider, 1988) : aux prises avec ce dernier problème d'optimisation, peu d'élèves ayant reçu un enseignement standard des dérivées lient le maximum du volume au point où la dérivée est nulle et, lorsqu'ils parlent de tangente, c'est pour évoquer une translation de l'axe Ox jusqu'à la hauteur où il frôle le maximum. Les chercheurs estiment que le contexte cinématique du premier problème peut aider les élèves à associer l'idée de maximum à l'annulation d'un taux de variation instantané, ici une vitesse. Quant au deuxième problème, il mobilise la même fonction que le premier. C'est en ce sens que le transfert est ici « sous contrat» car c'est cette variable didactique qui peut conduire les élèves à transférer de l'un à l'autre soit la réponse, soit la technique. L'enjeu est alors d'arriver à faire émerger l'idée que ces deux problèmes font partie de la même classe et ce qui nous intéresse ici c'est l'intelligibilité que les élèves expriment des résolutions de chacun des problèmes et de ce qu'elles ont en commun. La description ci-dessous montre le rôle joué par des jeux de langage dans la prise de conscience de certains d'entre eux.
Pour la plupart des élèves, il ne va pas de soi de rapprocher la recherche d'un maximum et l'annulation de la vitesse. Ceux qui en restent aux aspects graphiques ne parviennent qu'à une approximation numérique. Le professeur engage alors les élèves à interpréter le graphique en termes de mouvement :

P : «À l'instant qui vous intéresse, il avançait : il se met à reculer. Creusez ce que ça veut dire. »

Cette sollicitation guide l'un ou l'autre d'entre eux vers la solution :

M1 : «Calculer à partir de quand il commence à reculer. »

N1: «Alors attends: qu'est-ce que ça veut dire...

Ben qu'il a une vitesse positive...»

M1 : «Positive au début...»

N1: «Que la pente est positive et puis négative...»

M1 : «Ben il faudrait trouver un moyen de calculer à partir de quand, justement, il recule ; de voir par rapport à la vitesse qui est positive puis négative. »

M1 : «Il faut voir comment on a calculé les vitesses à partir des équations : il y a peut-être moyen de trouver l'équation de la vitesse. Enfin, tu vois, on avait à chaque fois trouvé une équation d'une vitesse. On avait... Tu vois, $3 \mathrm{t}^{2} \ldots 3 \mathrm{t}^{2}$ pour celle-là. »

M1 : «Donc, tout simplement plus grand que zéro, il n'y a pas moyen ? On dit tout simplement : quand [...]. Tu vois ? Ou bien, non : vitesse égale à zéro... Mais oui : elle augmente... Le moment où elle [la vitesse] sera égale à zéro, c'est le moment où elle [la distance] sera plus grande, parce ce qu'elle [la distance] augmente puis elle descend. »

N1: «Oui, c'est ça. »

E1 : «Oui, elle sera égale à zéro.»

C1: «Quand v est égale à 0 , oui. »

N1: « Oui, c'est ça, où la pente est nulle. »

Lorsque l'expérimentateur revient dans leur groupe, les élèves lui expliquent pourquoi ils ont écrit que la vitesse est nulle lorsque la distance est maximale :

E1 : $[\ldots]$ « Ici, on sait que la vitesse sera égale à zéro. [...] Parce que c'est... Il avance, il s'arrête, et puis il recule. »

Lorsqu'ils abordent le deuxième problème, les élèves prennent assez vite conscience d'une similitude formelle, voire d'une similitude graphique, entre les deux problèmes mais n'expriment pas forcément d'intelligibilité quant à la parenté de ceux-ci. Voici ce qu'en disent deux d'entre eux : 
Cl: « Je ne comprends pas comment on peut faire la même chose que l'autre fois vu qu'on n'a pas la vitesse, on n'a pas de vitesse ».

M1: «Il faut trouver une excuse pour avoir la vitesse $»$.

En revanche, quelques secondes plus tard, le même élève construit une procédure similaire à celle employée pour résoudre le premier en envisageant de faire jouer à $x$ et $\Delta x$ le même rôle qu'à $t$ et $\Delta t$ précédemment :

M1 : « Mais, au lieu de dire... On ferait mieux de dire $\mathrm{x}+\Delta \mathrm{x}$. [...] Mais oui mais la vitesse [...] On peut utiliser qu'on avait $\mathrm{t}+\Delta \mathrm{t}$ avant, pour trouver un moment et un moment juste après. Mais là, on peut utiliser la même chose pour dire un volume précis et un volume après. Donc, $\mathrm{x}+\Delta \mathrm{x}$, enfin, je ne sais pas...»

Enfin, un élève observe l'intérêt d'avoir proposé les deux problèmes dans cet ordre :

E1 : «Si on avait commencé par ça [le problème du volume], ça aurait été plus compliqué, disons. Parce que, si on commençait par ça, on ne pouvait pas égaliser cette équation [avec la vitesse instantanée], dire qu'elle était nulle. Donc on n'arrivait pas à trouver cette réponse-là ».

Rappelons l'enjeu majeur de ces deux problèmes : instituer la classe des problèmes d'optimisation à partir de deux d'entre eux, le premier se situant dans un contexte graphico-cinématique, le second faisant partie du cadre géométrique. Comme observé, il ne va pas de soi qu'un problème de distance maximale peut se penser en termes de vitesse nulle. Les élèves s'en tiennent d'abord à des aspects graphiques jusqu'au moment où le professeur commente le mouvement rectiligne représenté par ce graphique en parlant d'un mobile qui «avance » ou " recule ». C'est là un langage qui situe l'expérience dans un univers encore peu épuré : on aurait pu, en faisant référence à un mobile ponctuel qui n'a ni avant ni arrière, parler d'un mouvement dans le sens positif ou négatif sur la trajectoire orientée. Mais c'est un discours qui fait mouche puisque les élèves s'en emparent pour l'interpréter en termes de vitesse positive ou négative, ou encore de pente positive ou négative, conformément à ce qui a été enseigné auparavant. Et c'est ce qui permet à l'un d'eux de convoquer un type de calcul déjà pratiqué dans des situations antérieures et qu'il appelle "l'équation de la vitesse ». Toutefois, il faut remarquer que penser à annuler cette vitesse ne lui vient pas directement à l'esprit car il semble envisager tout d'abord de résoudre une inéquation : « tout simplement plus grand que $0 »$. Par contre, une fois exprimée (par un autre) cette idée d'annulation, tous les élèves s'en emparent ; l'un d'eux l'interprète en termes d'annulation de la pente et un autre la justifie en revenant au mobile qui « s'arrête » entre le moment où il « avance » et celui où il « recule». On voit ici s'entremêler plusieurs jeux de langage, le discours portant dialectiquement sur le mouvement d'un mobile dans un langage proche du quotidien, sur des savoirs physiques déjà construits comme le signe de la vitesse ou son annulation, sur des connaissances graphiques ou symboliques qui les traduisent: la pente, une inéquation ou une équation. On peut donc conclure que l'univers graphico-cinématique dans lequel les élèves ont été plongés antérieurement a pu constituer, pour ce groupe d'élèves au moins, un univers cognitif et langagier permettant une certaine dialectique avec un système d'écritures symboliques.

Comme on l'a dit plus haut, le deuxième problème est d'un point de vue formel équivalent en tout point au premier : même fonction mobilisée, même résolution via un calcul de dérivée et son annulation. Ce pourrait être suffisant pour que les élèves copient la réponse de l'un à l'autre. Ils n'en font rien, même si l'un d'eux est conscient que c'est à dessein que le professeur leur propose les problèmes dans cet ordre. Au-delà de l'unité fonctionnelle, il faut ici comprendre qu'une même technique permet de les résoudre tous deux. La difficulté est qu'il faut penser alors à une sorte de pendant de la vitesse dans le deuxième problème, sans pouvoir encore parler de dérivée. Il convient de souligner que le mot même de " dérivée » est significatif d'un autre niveau d'appréhension de ces divers problèmes et atteste que l'on est conscient de les résoudre en cherchant une nouvelle fonction, « dérivée » d'une autre au moyen des règles de dérivation. Mais les élèves concernés n'ont pas encore appris de calcul de dérivées et cherchent, comme l'un d'eux l'exprime, « une excuse pour avoir la vitesse ». À défaut de pouvoir s'appeler "dérivée ", l'équivalent de la vitesse dans le problème de la boîte aurait pu être ce que certains nomment le " taux instantané de variation». Effectivement, le taux est un rapport tout comme la vitesse ; comme celle-ci, il mobilise une variation : 
ici variation d'un volume au lieu d'une variation de position ; le qualificatif « instantané », par contre, peut paraître incongru dans le problème du volume étant donné que, contrairement au problème de la vitesse, la variable indépendante n'est plus le temps. Bref, ce taux n'est pas plus précisément défini que la vitesse, qui porte un sens et des jeux de langage plus anciens et riches. Car, comme Schneider (1988) l'a observé, les élèves qui parviennent à injecter une idée de variation là où elle ne va pas de soi a priori le font souvent en référence au temps de déroulement de la pensée. Il est symptomatique de voir comment l'élève Ml subodore l'intérêt de remplacer $\mathrm{t}+\bigotimes \mathrm{t}$ par $\mathrm{x}+\nabla \mathrm{x}$ en faisant le parallèle entre, d'une part, « un moment et un moment juste après » et, d'autre part, « un volume précis et un volume après » : c'est bien $\mathrm{x}+\nabla \mathrm{x}$ qu'il note mais c'est bien « après » qu'il dit. On peut donc penser, au niveau du type de calculs considérés à ce stade (qui consistent, rappelons-le, à supprimer des termes contenant $\nabla \mathrm{x}$ dans un taux

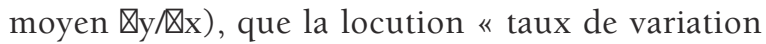
instantané » facilite la prise de conscience des liens entre les deux problèmes étudiés ici. Même si elle peut favoriser une confusion chez les élèves, entre variation temporelle et variation en fonction d'une variable indépendante quelconque, confusion qui nécessitera de la part du professeur un discours d'explicitation sur la signification du remplacement de $t$ en $x$. On trouve, dans cet épisode didactique, tout l'intérêt d'un jeu de langage approprié pour faire vivre un système de notations donnant prise à une technique nouvelle.

Sur base d'expériences diverses, Mercier (2008) montre l'importance, dans la gestion de la classe, de jeux de langage susceptibles de produire des significations nouvelles. Et ce, que cette gestion soit de l'ordre de l'accompagnement, le professeur aidant " les élèves à nommer et désigner les objets du milieu » et les aidant "à agir sur ces objets », ou de l'ordre de l'analyse lorsque le professeur « identifie dans l'action des élèves des éléments 'pertinents'ou des éléments 'contradictoires' « avec les objets de savoir enjeux de la séance ». Ces jeux de langage sont rendus possibles par la « disponibilité d'un lexique adéquat » ou, plus généralement, celle d'une « dialectique entre une notation ou un système de notations et une notion ou un univers cognitif associés ». Ils permettent et supposent à la fois des 'formes de vie particulières'qui sont « les pratiques d'un collectif de pensée (Fleck, 1935/2005), cherchant à partager notions et notations pour la résolution d'une classe de problèmes $»$. C'est bien ce que montre l'expérience relatée ici, sur base de quelques propos d'élèves qui peuvent suggérer ce que devrait contenir le discours du professeur lorsque celui-ci institue la classe des problèmes d'optimisation à partir des deux spécimens proposés aux élèves.

Dans l'hypothèse d'un enseignement qui vise à mettre en évidence, d'entrée de jeu, les questions auxquelles les savoirs mathématiques apportent une réponse, le professeur doit développer avec ses élèves des rapports au savoir d'un type nouveau. Mais deux manières contrastées d'agir délimitent l'espace de choix du professeur.

D'un côté, la recherche de situations fondamentales, qui peuvent être déclinées en jeux dévolus aux élèves, au sens de Brousseau (1997) ; nous avons en effet proposé (Schneider et Mercier, 2008) de reprendre l'idée originale de Brousseau qui nommait « jeux » les situations adidactiques et « situations » les organisations institutionnelles se déclinant en suites de jeux définissant des milieux d'action sous contrat. Cela réserve à l'analyse que peut faire un observateur la démonstration du fait que l'action dans un jeu soit effectivement adidactique. Nous obtenons ainsi une version non prescriptive de la TSD, en réservant à l'analyse la démonstration du fait que l'action dans un jeu est effectivement adidactique.

Mais de l'autre côté, le professeur peut aussi organiser une rencontre des savoirs qui soit culturelle-mimétique au sens de Chevallard (1999) ; cela consiste « à expliciter - sur le mode discursif - les raisons d'être du savoir ainsi rencontré ». Chevallard a développé cette idée en montrant que l'enquête sur un savoir comme réponse à la nécessité d'agir dans une situation problématique est une clé du renouvellement des praxéologies didactiques.

Nous affirmons ici que les deux positions doivent être tenues. Des suites de jeux permettent la production, par les élèves organisés en collectif de recherche, des savoirs dont la consistance doit être explorée ; d'autres suites de jeux organisent une enquête sur des savoirs dont l'efficacité doit être explorée. Ce sont les deux temps de toute étude solide d'un domaine de réalité et des œuvres qui lui sont associées. La notion de jeu (un jeu d'apprentissage) devient alors centrale dans l'analyse didactique (Sensevy et Mercier, 2007).

Dans les deux cas, le savoir est effet du rapport à un milieu, organisé comme un jeu d'apprentissage, qui l'a fait naître comme connaissance. Le jeu d'apprentissage 
s'intègre d'abord dans une situation de référence où l'élève peut saisir, au-delà des actions réalisées ou des discours proposés, ce qu'il y a à comprendre c'est-àdire l'efficacité du savoir, mais aussi bien les orientations stratégiques que propose la connaissance. Il s'intègre dans une suite de jeux d'apprentissage où l'élève peut identifier la classe de problèmes que les procédures engagées permettent de résoudre, le domaine de variabilité que la classe définit et les démarches attendues de lui à ce propos, dans l'institution scolaire. L'organisation de ces mouvements de l'étude est de la responsabilité du professeur qui, ce faisant, enseigne : leur description met en évidence la difficulté du métier et dénonce les mots d'ordre simplistes pour ce qu'ils sont.

\section{CONCLUSION}

Les discours officiels sur l'APC font l'apologie, en mathématiques ou ailleurs, des « situationsproblèmes » et de la "résolution de problèmes », sans être explicites sur ce qu'il faut entendre par là. Les chercheurs en éducation oscillent entre deux idées contraires : d'un côté, la résolution de problèmes ne s'enseigne pas; de l'autre, elle suppose un enseignement méthodologique axé sur des aspects transversaux. Or, en mathématiques mais sans doute aussi dans d'autres domaines de savoir, les problèmes sont groupés en classes, les savoirs et techniques associées étant les éléments majeurs de cette classification. En ce sens, la didactique est outillée pour répondre à la demande sociale qui s'exprime au travers de la réforme "des compétences " et qui demande que les savoirs enseignés soient bien, pour les élèves, pouvoirs d'agir en situation : compétence. Mais elle interroge à la fois les formes de réalisation de cette demande, qui n'ont pas conduit à identifier ni les situations (les problèmes et les questions contextuels) définissant les compétences attendues, ni les formes scolaires (celles qui sont montrées en exemple, en un mot, ne permettent pas au professeur d'enseigner).

Les classes de problèmes étudiées dans un enseignement traditionnel relèvent en principe d'un type bien particulier d'organisation de savoirs, ce que l'on appelle « une discipline d'enseignement » et l'organisation des savoirs en disciplines fonde aujourd'hui encore les curriculums. L'évolution de l'enseignement que nous avons envisagée ne suppose pas cette organisation, qui a déjà volé en éclats dans la plupart des enseignements professionnels ou techniques. Il suffit que l'organisation d'une suite de jeux d'apprentissage permette d'identifier explicitement des savoirs qui seront les cibles officielles de l'étude. Cela semble une condition faible mais cela désigne la plus grande difficulté des ingénieries didactiques qui développent une intervention sur plus de quelques séances.

C'est la condition pour que le professeur puisse diriger ce processus, selon les techniques génériques dont nous avons montré un exemple en mathématiques. Sous cette condition, les savoirs pourraient être aussi « des disciplines de l'action » et porter sur des domaines de réalité et d'action identifiés, comme peuvent l'être les ingénieries et plus largement ce que l'on nomme les « savoirs professionnels ». Nous insistons sur ces questions et nous y revenons ici, parce que nous pensons que le succès du mot d'ordre « enseigner par compétences pour former des compétences » ne tient pas seulement aux économies qu'il fait miroiter auprès des décideurs, mais sans doute aussi au fait qu'il semble proposer une solution aux problèmes que pose l'organisation scolaire de la transmission des œuvres culturelles. Dans les écoles, les étudiants en effet apprennent efficacement des savoirs, quand la société voudrait que les apprentissages garantissent leur compétence : ce qui d'ordinaire s'acquiert d'expérience, dans un tout autre temps. Ces problèmes sont désignés par les théories didactiques sous le terme générique de " effets de la transposition didactique » et les réformes actuelles semblent viser à en diminuer les effets, sans que pourtant le but n'ait été explicité : comme si les discours sur la nécessité des savoirs, au fondement de la formation de l'expérience, ne suffisaient plus à justifier du coût de l'école lorsque l'école devient " école pour tous » et développe des enseignements pour toute une classe d'âge, sur plus de dix ans.

Une synthèse de résultats empruntés tant à la didactique des mathématiques qu'à la psychologie cognitive (Schneider, 2006b) inspire alors un mode d'enseignement de la résolution de problèmes où l'on apprend aux élèves, d'une part, à gérer la variabilité des classes de problèmes, savoir par savoir et, d'autre part, à brasser à la fois plusieurs classes de problèmes, ce qui suppose le choix de savoirs appropriés. Dans un tel enseignement, fédérer quelques problèmes parents autour d'un même savoir ou d'une même technique, constitutive de la définition 
même des savoirs, est une démarche particulièrement délicate. Dans cette perspective, la désignation des savoirs suppose, comme on a pu le voir dans le cas exemplaire du jeu entre les deux problèmes de variation, des jeux de langage qui enclenchent une dialectique entre des notions et des notations. Une telle approche constitue, à nos yeux, un antidote à ce qu'on peut observer dans les manuels comme dans les classes : une liste d'activités suivie d'une synthèse qui fait l'économie du lien entre les deux parties. Elle apporte également un éclairage nouveau et complémentaire sur d'autres recherches portant sur la résolution de problèmes, ses « enjeux non explicités d'apprentissage » (Castela, 2007 et 2008) et la portée du « levier méta ( Robert et Tenaud, 1988). Nous savons dorénavant le prix qu'il faut payer, si l'on veut éviter un déficit « d'intelligibilité disciplinaire » et un malentendu scolaire propre à accentuer des inégalités scolaires au sens développé par Bautier et Rayou (2009), le projet d'enseignement n'étant visible qu'aux élèves qui peuvent l'appréhender en dehors des temps et lieux scolaires.

La question de l'enseignement devient ainsi double, et nous pensons qu'elle n'est pas posée aux seuls tenants des mathématiques, mais à tous les membres de la noosphère quelle que soit leur discipline ou matière de référence :

1) Quelles classes de problèmes étudier ?

2) Quelles organisations de systèmes sémiotiques privilégier?

Nous avons ici travaillé la première, la seconde relève des recherches sur l'introduction à des pratiques algébriques (par exemple: Krysinska, Mercier, Schneider, 2009) ou géométriques et nous la signalons seulement. Comme ces questions se poursuivent d'année en année, domaine d'étude par domaine d'étude, le travail pédagogique de définition d'un curriculum didactiquement cohérent et socialement acceptable est un chantier politique et technique qui devrait être ouvert faute de quoi, les réformes des systèmes d'enseignement sont et demeureront pour les professeurs la source d'injonctions paradoxales paralysantes.

\section{NOTES}

1. Ce travail est un des résultats du séjour de Maggy Schneider à Marseille, comme professeur invité par l'INRP, auprès de l'UMR P3, en avri let mai 2010. Depius, l'INRP est devenu l'IFE (Institut Français de l'Éducation) institut interne de l'ENS-Lyon et par voie de conséquence, l'UMR P3 est devenue Équipe d'Accueil Miste (EAM).

2. La CFWB est englobée depuis dans la CWB: Communauté Wallonie-Bruxelles.

3. Un état paradoxal puisque le retour aux savoirs comme pouvoir d'agir avait justifié l'Encyclopédie de d'Alembert et Diderot, et avait inauguré l'organisation des savoirs en disciplines contre l'organisation ancienne de l'université médiévale. Le «monumentalisme » dénoncé par Chevallard est une possible raison : les savoirs deviennent « monuments » à visiter sans que leur raison d'être ne soit explicitée.

4. Ernst Mach (1838-1916) physicien et philosophe autrichien. 


\section{RÉFÉRENCES}

Bautier, E., Rayou P. (2009), Les inégalités d'apprentissage, Paris : PUF.

Baechler, J. (1976), Qu'est-ce que l'idéologie ?, Paris : Gallimard.

Beckers, J. (2002), Développer et évaluer des compétences à l'école: vers plus d'efficacité et d'équité, Bruxelles : Éditions Labor.

Brousseau, G. (1973). «Peut-on améliorer le calcul des produits de nombres naturels ? ". Actes du $3^{e}$ congrès des sciences de l'éducation «Apports des disciplines fondamentales aux sciences de l'éducation $»$ tome 1 .

Brousseau, G. (1986). Fondements et méthodes de la didactique des mathématiques. Recherches en Didactique des Mathématiques, 7 (2), 33-115.

Brousseau, G. (1998), La théorie des situations didactiques, Grenoble : La Pensée Sauvage.

Castela, C. (2007), Travail de la question des enjeux non explicités d'apprentissage avec les outils de la théorie anthropologique. Curriculum et chronogenèse praxique. In Ruiz-Higueras et Al. (Eds.), Sociedad, escuela y matemáticas. Aportaciones de la Teoría Antropológica de lo Didáctico (TAD) (p. 117-138). Jaén : publicaciones de la Universidad de Jaén.

Castela, C. (2008). Travailler avec, travailler sur la notion de praxéologie mathématique pour décrire les besoins d'apprentissage ignorés par les institutions d'enseignement. Recherches en Didactique des Mathématiques, 28 (2), 135-182.

Castella, C., Mercier, A. (1995). Peut-on enseigner des méthodes ? Comment les élèves apprennent-ils des méthodes ? Petit x, 41, 5-25.

Castella, C., Mercier, A. (1994). Peut-on enseigner des méthodes ? Comment les élèves apprennent-ils des méthodes ? Bulletin de la Commission Inter-IREM de didactique des mathématiques, 1.

Chevallard, Y. (1990). Le passage de l'arithmétique à l'algèbre dans l'enseignement des mathématiques au collège. Troisième partie, Voies d'attaque et problèmes didactiques. Petit X, 19, 43-72.

Chevallard, Y. (1991), La transposition didactique, Grenoble: La Pensée Sauvage.

Chevallard, Y. (1992). Concepts fondamentaux de la didactique: perspectives apportées par une approche anthropologique. Recherches en Didactique des Mathématiques, 12 (1), 72-112.

Chevallard, Y. (1999). Lanalyse des pratiques enseignantes en théorie anthropologique du didactique. Recherches en Didactique des Mathématiques, 19 (2), 221-265.

Crahay, M. (2006). Dangers, incertitudes et incomplétude de la logique par compétences en éducation. Revue Française de Pédagogie, 154, 97-110.

Crahay, M., Forget, A. (2006). Changements curriculaires : quelle est l'influence de l'économique et du politique? In F. Audigier, M. Crahay, J. Dolz, \& A. Delhaxhe (Éds.), Curriculum, enseignement et pilotage. Bruxelles : De Boeck.
De Ketele, J.-M. (2003). Approche socio-historique des compétences dans l'enseignement. In Chr. Bosman, F.-M. Gerard \& X. Roegiers (2001) Quel avenir pour les compétences ?, Bruxelles : De Boeck, 83-92.

Douady, R. (1986). Jeux de cadres et dialectique outil-objet Recherches en Didactique des Mathématiques, 7 (2), 5-31.

Draelants, H., Dupriez, V., Maroy, Chr. (2003), Le système scolaire en Communauté française, Bruxelles : CriSP.

Fagnant, A., Demonty, I. (2005), Résoudre des problèmes : pas de problème! Guide méthodologique et documents reproductibles, Bruxelles: De Boeck.

Fleck, L. (1935/2005), Genèse et développement d'un fait scientifique, Paris : Les belles lettres.

Gantois, J.-Y., Schneider, M. (2012). Une forme embryonnaire du concept de dérivée induite par un milieu graphico-cinématique dans une praxéologie « modélisation ». Recherches en Didactique des Mathématiques, 32 (1), 56-100.

Gerard, F.-M., Van Lint-Muguerza, S. (2003). Quel équilibre entre une appréciation globale de la compétence et le recours aux critères ? In Chr. Bosman, F.-M. Gerard \& X. Roegiers (Éds.), Quel avenir pour les compétences? (p. 135-140). Bruxelles: De Boeck.

Jonnaert, P, Vander Borght, C. (1999), Créer des conditions d'apprentissage, Bruxelles: De Boeck Université.

Kahn, S. (2010). Différents types de compétences : Comment les faire acquérir? Comment les évaluer ? Socle commun et travail par compétences. Balises et boussole. http://tice.ac-toulouse.fr/automne_modules_files/ documents/public/r660_27_cahiers_pedagogiques_ socle_commun_et_travail_par_competences_-_balises_et_boussole.pdf

Krysinska, M., Mercier, A. \& Schneider, M. (2009). Problèmes de dénombrement et émergence de premiers modèles fonctionnels. Recherches en Didactique des Mathématiques, 29 (3), 247-304.

Lalande, A. (2002). Dictionnaire de philosophie, Paris : PUF. Mach, E. (1925), La mécanique, Paris : Hermann.

Mercier (2008). Questions d'épistémologie des situations. Conférence introductive au Colloque international de l'AFIRSE.

Perkins, D., Salomon, G. (1989). Are Cognitive Skills Context-Bound ? Educational Researcher, 17, 16-25.

Reusser, Stebler (1997). Every word problem has a solution,. The social rationnality of mathematical modelling in schools. Learning and Instruction, 7 (4), 309327.

Rey, B. (2009). Les compétences, oui, mais ce qui compte, c'est de faire apprendre... Café pédagogique du 6 décembre 2009, INRP.

Robert, A., Tenaud, I. (1988). Une expérience de la géométrie en terminale C. Recherches en Didactique des Mathématiques, 9 (1), 31-70.

Robert, A., Robinet, J. (1996). Prise en compte du méta en didactique des mathématiques. Recherches en Didactique des Mathématiques, 16 (2), 145-176. 
Roegiers, X. (2000). Une pédagogie de l'intégration. Compétences et intégration des acquis dans l'enseignement, Bruxelles : De Boeck Université.

Schneider, M. (1988). Des objets mentaux « aire » et « volume " au calcul des primitives. Dissertation doctorale, université catholique de Louvain.

Schneider, M. (2004). Viser le «transversal» à travers du « bon disciplinaire » ou trois compétences transversales contextualisées au sein de l'enseignement des mathématiques. Repères-IREM, 55, 51-70.

Schneider, M. (2006a). Quand le courant pédagogique « des compétences » empêche une structuration des enseignements autour de l'étude et de la classification de questions parentes. Revue Française de Pédagogie, 154, 85-96.

Schneider, M. (2006b). Comment des théories didactiques permettent-elles de penser le transfert en mathématiques ou dans d'autres disciplines ? Recherches en Didactique des Mathématiques, 26 (1), 9-38.

Schneider, M. (2007). Les compétences comme cadre pour organiser des enseignements de mathématiques? Oui, mais... Quelques dérives possibles. Canadian Journal of Science, Mathematics and Technology Education, 7 (1), 28-40.

Schneider, M. (2008), Traité de didactique des mathématiques. La didactique par des exemples et contre-exemples, Les Éditions de l'université de Liège.

Schneider, M. (2011a). Mise en œuvre de l'approche par compétences en Communauté française de Belgique : ce que la recherche en didactique des mathématiques aurait pu ou pourrait apporter. In J. Lebeaume, A. Hasni \& I. Harlé (Éds.), Recherches et expertises pour l'enseignement de la technologie, des sciences et des mathématiques. Louvain-la-Neuve : De Boeck Université.
Schneider, M. (2011b). Ingénieries didactiques et situations fondamentales. Quel niveau praxéologique? In C. Margolinas, M. Abboud-Blanchard, L. Bueno-Ravel, N. Douek, A. Fluckiger, \& P. Gibel, En amont et en aval des ingénieries didactiques. Grenoble: La Pensée Sauvage.

Schneider, M. (2012). Un obstacle épistémologique comme trait d'union des travaux d'un laboratoire de didactique des mathématiques. In S. Coppé \& M. Haspekian, Actes du Séminaire national de Didactique des Mathématiques 2010-2012. Paris: IREM de Paris 7.

Schneider, M., Mercier, A. (2008). Situation adidactique, situation didactique, situation-problème : circulation de concepts entre théorie didactique et idéologies pour l'enseignement. Actes du Colloque «Didactiques: quelles références épistémologiques ? », organisé à Bordeaux, en mai 2005.

Schönfeld, A.H. (1989). Teaching mathematical thinking and problem solving. In L.B. Resnick \& L.E. Klopfer (dir.), Toward the thinking curriculum: Current cognitive research (p. 83-104). Alexandria : VA : Association for Supervision and Curriculum Development.

Schubauer-Leoni, M-L. (1988). « Le contrat didactique dans une approche psycho-sociale des situations d'enseignement ». Interactions didactiques, $\mathrm{n}^{\circ} 8$, universités de Genève et de Neuchâtel, 63-75.

Sensevy, G., Mercier, A. (2007). Agir ensemble. Rennes : Presses universitaires de Rennes.

Tardif, J. (1999). Le transfert des apprentissages, Montréal : Les Éditions Logiques.

Verschaffel, L., Greer, B., De Corte, E. (2000), Making sense of word problems, Lisse, The Netherlands : Swets $\&$ Zeitlinger. 